\title{
Hyperoxia Exposure Alters Hepatic Eicosanoid Metabolism in Newborn Mice
}

\author{
LYNETTE K. ROGERS, TRENT E. TIPPLE, RODNEY D. BRITT, AND STEPHEN E. WELTY
}

Department of Pediatrics, The Ohio State University, Columbus, Ohio 43205

\begin{abstract}
Prematurely born infants are often treated with supraphysiologic amounts of oxygen, which is associated with lung injury and the development of diseases such as bronchopulmonary dysplasia. Complimentary responses between the lung and liver during the course of hyperoxic lung injury have been studied in adult animals, but little is known about this relationship in neonates. These studies tested the hypothesis that oxidant stress occurs in the livers of newborn mice in response to continuous hyperoxia exposure. Greater levels of glutathione disulfide and nitrotyrosine were detected in lung tissues but not liver tissues from newborn mice exposed to hyperoxia than in room air-exposed controls. However, early increases in 5-lipoxygenase and cyclooxygenases-2 protein levels and increases in total hydroxyeicosatetraenoic acid and prostaglandin levels were observed in the liver tissues of hyperoxia-exposed pups. These studies indicate that free radical oxidation occurs in the lungs of newborn pups exposed to hyperoxia, and alterations in lipid metabolism could be a primary response in the liver tissues. The findings of this study identify possible new mechanisms associated with hyperoxic lung injury in a newborn model of bronchopulmonary dysplasia and thus open opportunities for research. (Pediatr Res 67: 144-149, 2010)
\end{abstract}

$\mathrm{P}$ remature birth is the leading cause of infant mortality and morbidity in the United States (1-3). Although oxygen and mechanical ventilation are essential therapies for the treatment of premature infants, they are closely associated with lung injury and the development of diseases such as bronchopulmonary dysplasia $(4,5)$. Mechanistically, the development of bronchopulmonary dysplasia includes inflammatory and oxidative responses that ultimately lead to a deficit in lung development (6-8).

Systemic inflammatory responses subsequent to injury in a single organ are well documented. Frequently, lung injury affects liver function, and hepatic events elicit alterations in lung function. An example of hepatic and pulmonary intercommunication is hepatopulmonary syndrome, which is characterized by vasodilatation in the lung in response to liver trauma (9). Although the mechanisms of injury are not completely understood, the leading hypothesis is that molecules released by the liver during injury enter the bloodstream and initiate responses in the lung. Excessive pulmonary production

Received June 9, 2009; accepted September 15, 2009.

Correspondence: Lynette K. Rogers, Ph.D., Center for Perinatal Research, Department of Pediatrics, The Research Institute at Nationwide Children's Hospital, Ohio State University, 700 Children's Dr., Columbus, Ohio 43205; e-mail: Lynette. Rogers@NationwideChildrens.org

Supported by American Thoracic Society and The Research Institute at Nationwide Children's Hospital. of $\mathrm{NO}$ and other molecules such as tumor necrosis factor $\alpha$ and heme oxygenase- 1 have also been identified as responses to hepatic injury (10). Consequently, the relationship and complimentary responses between the lung and the liver are being actively studied in adults (11-17) but little is known about such a relationship in neonates.

The studies described in this report were designed to test the hypothesis that oxidant stress occurs in the liver of newborn mice in response to hyperoxia exposure. Enhanced understanding of organ-specific responses to hyperoxia that could contribute to the pathogenesis of newborn hyperoxic lung injury will identify additional pathways for research. These discoveries should enable the development of novel therapies directed at the prevention of lung injury in premature neonates.

\section{METHODS}

Animal models. Animal study protocols were approved by the Institutional Animal Care and Use Committee at The Research Institute at Nationwide Children's Hospital. Newborn pups were randomized and equally distributed between $\mathrm{C} 3 \mathrm{H} / \mathrm{HeN}$ dams delivering litters within a 12 -h period. Half of the dams with litters were placed in a Plexiglas chamber containing a $10-\mathrm{L} / \mathrm{min}$ flow of $>95 \%$ (daily average $98 \%$ ) $\mathrm{O}_{2}$, whereas the other half were placed in room air (RA) within the first $12 \mathrm{~h}$ of life. The dams were rotated daily between hyperoxia and RA to prevent oxygen toxicity, whereas the pups were maintained in continuous exposure. Twenty-four hours of hyperoxia exposure was designated as $\mathrm{d} 1$. On 1, 3, 7, or $14 \mathrm{~d}$ of life the pups were euthanized by intraperitoneal injections of $200 \mathrm{mg} / \mathrm{kg}$ of sodium pentobarbital, and the tissues were harvested. The tissue size and fragility prevented perfusion so subsequent analyses were all performed on nonperfused tissues.

Glutathione and glutathione disulfide. Glutathione (GSH) contents were measured in $10 \%$ tissue homogenates prepared in $50 \mathrm{mM} \mathrm{NaPO}, 50 \mathrm{mM}$ serine borate, $17.5 \mathrm{mM}$ EDTA, pH 7.4. Glutathione disulfide (GSSG) levels were measured in $N$-ethyl maliemide-treated supernatants. Tissues were homogenized, as described earlier, with the addition of $10 \mathrm{mM} \mathrm{N}$-ethyl maliemide to the buffer. GSSG homogenates were separated by solid phase extraction, and the eluent was used for GSSG measurements. Tissue contents of both GSH and GSSG were measured by the enzyme recycling method described by Rogers and Smith (18).

Nitrotyrosine. Nitrotyrosine levels were measured by slot-blot technique. A standard curve was generated using increasing amounts of tetranitromethane-treated albumin (19). One to two micrograms of protein was loaded into each well, and each sample was run in duplicate. The proteins were absorbed onto nitrocellulose membranes and were probed with anti-nitrotyrosine primary [(Upstate) Millipore, Inc, Billerica, MA] and anti-mouse secondary antibodies (1:12000, BioRad, Hercules, CA). The membranes were visualized by enhanced chemiluminescence detection (GE Healthcare Biosciences Corp, Piscataway, NJ), and band densities were quantitated by densitometry using Image Quant software, version 5.0 (Molecular Dynamics, Sunnydale, CA),

\footnotetext{
Abbreviations: RA, room air; CoASH, coenzyme A; CoASSG, coenzyme A glutathione disulfide; COX, cyclooxygenase; GSH, glutathione; GSSG, glutathione disulfide; HETE, hydroxyeicosatetraenoic acid; LO, lipoxygenase
} 

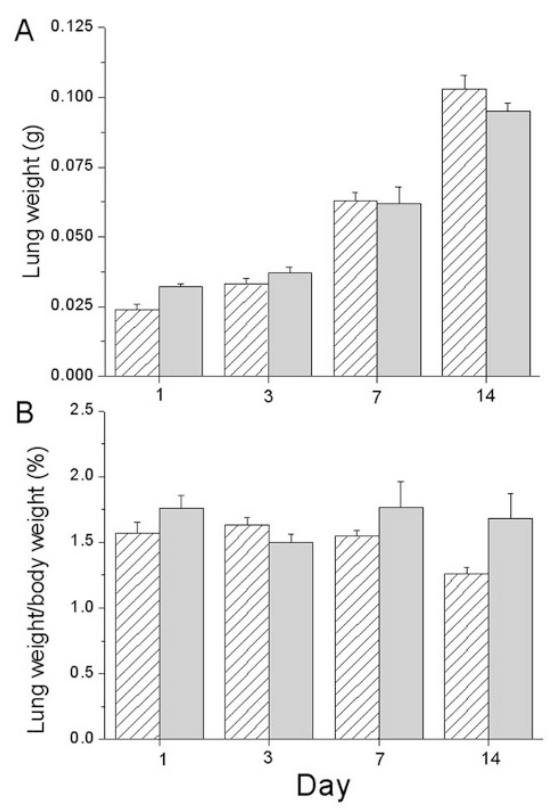

Figure 1. Lung and liver weights in newborn $\mathrm{C} 3 \mathrm{H} / \mathrm{HeN}$ pups. Pups were exposed to $1,3,7$, or $14 \mathrm{~d}$ of RA (white hatched bars) or $>95 \% \mathrm{O}_{2}$ (gray bars). Pups were killed at the designated times. Total lung and liver weights were recorded $(A$ and $C$ ). Ratios of lung weight/body weight and liver weight/body weight were calculated $(B$ and $D)$. Results are reported as mean \pm SEM and data were assessed by twoway ANOVA with modified $t$ test posthoc for individual differences, $n=10$ to $18, p<0.05$. An effect of day was observed on lung weights and lung weight/body weight ratios. An effect of day, an effect of exposure, and an interaction were observed on liver weights with an effect of day on the liver weight/body weight ratios. *Indicates different from same day RA.

and values were calculated using an experimentally derived standard curve for each membrane.

CoA (CoASH) and CoA glutathione disulfide. CoASH and CoA glutathione disulfide (CoASSG) contents were determined by $\operatorname{HPLC}(18,20,21)$. The CoA species were separated on a Zorbax SB-C $\mathrm{C}_{18}$ column using the mobile phases: A) $25 \%$ methanol, $75 \%$ water; B) $65 \%$ methanol, $35 \%$ water; C) $0.1 \mathrm{M}$ tetrabutylammonium hydrogen sulfate, $\mathrm{pH}$ 5.0. The gradient was 2 min at $90 \% \mathrm{~A}, 0 \% \mathrm{~B}, 10 \% \mathrm{C}$, followed by a 20 -min linear gradient to $5 \% \mathrm{~A}$, $85 \%$ B, $10 \%$ C. UV detection at $254 \mathrm{~nm}$ was used for quantitation. Samples were analyzed using experimentally derived standard curves.

Western blots. Frozen lung or liver tissues were homogenized, and proteins $(25-50 \mu \mathrm{g})$ were separated by $12 \%$ SDS-PAGE (Invitrogen, Carlsbad, $\mathrm{CA}$ ) and transferred to polyvinylidene fluoride membranes. Membranes were probed with antibodies to cyclooxygenases (COX)-2 (rabbit anti-mouse, 1:200, Abcam, Cambridge, MA), 5-lipoxygenase (LO) (mouse monoclonal, 1:1000, BD Bioscience, San Jose, CA), and HRP-conjugated rabbit antimouse secondary antibody (1:12000, BioRad, Hercules, CA). Blots were assessed with ECL detection (GE Healthcare Biosciences Corp, Piscataway, $\mathrm{NJ}$ ), and expression levels were quantitated by densitometry using Image Quant software, version 5.0 (Molecular Dynamics, Sunnydale, CA). The density of the band for the protein of interest was normalized to the density of $\beta$-actin protein (mouse monoclonal 1:5000, Abcam; goat anti-mouse, 1:15000, BioRad, Hercules, CA).

Lipid analyses. Lung tissues were homogenized and each sample was spiked with an internal standard solution (deuterated standards from each lipid group) then extracted using the Bligh-Dyer technique. Eicosanoids were analyzed by LC/MS/MS on an Applied Biosystems 4000 QTrap equipped with a Shimadzu HPLC. Separation was achieved using a Zorbax SB-C18 column, a flow rate of $0.3 \mathrm{~mL} / \mathrm{min}$, and a gradient of $8.3 \mathrm{mM}$ acetic acid, $\mathrm{pH}$ 5.7 (mobile phase A) and acetonitrile:2-propanol (50:50) (mobile phase B) as follows: 3 min hold at $15 \% \mathrm{~B}, 10$ min linear to $55 \% \mathrm{~B}, 15$ min linear to $80 \%$ B. The samples are analyzed in negative ionization mode using multiple reaction monitoring. Individual calibration curves were generated for each group of analytes, and sample concentrations were calculated using isotope dilution corrections.

Statistics. Data collected from analyses were analyzed by two-way ANOVA with day of life and oxygen exposure as independent variables. When a two-way ANOVA indicated a significant difference, individual differences were detected with posthoc testing using modified $t$ tests, $p<$ 0.05. All analyses were performed with SPSS Windows version 15.0 (Chicago, IL).

\section{RESULTS}

This data indicate that lung weights increase developmentally in both exposure groups with no effect of hyperoxia exposure detected (Fig. 1A) and no differences in lung weight/ body weight ratios (Fig. 1B). Liver weights were not different between RA and $>95 \% \mathrm{O}_{2}$ exposed mouse pups at $\mathrm{d} 1,3$, or 7 , however, at d 14 the liver weights were lower in the $>95 \%$ $\mathrm{O}_{2}$-exposed pups than in control pups (Fig. 1C). These lower liver weights were proportional to lower overall body weights as indicated by the fact that liver weight to body weight ratios were not different at d 14 (Fig. 1D).

GSH and GSSG levels were measured in lung and liver tissues from pups exposed to RA or $>95 \% \mathrm{O}_{2}$ as an assessment of peroxide-mediated oxidation. Our analyses of lung GSH levels indicated an effect of hyperoxia exposure, an effect of day and an interaction between exposure and day. Lung GSH levels were greater in the $>95 \% \mathrm{O}_{2}$-exposed pups than in the RA-exposed pup tissues at $\mathrm{d} 7$ and 14 (Fig. 2A). Furthermore, our analyses indicated an effect of hyperoxia exposure on lung GSSG levels with higher levels detected in the $>95 \% \mathrm{O}_{2}$-exposed pups than in the RA-exposed pups at $\mathrm{d}$ 3, 7, and 14 (Fig. 2B). Interestingly, hepatic GSH levels were substantially lower in the $>95 \% \mathrm{O}_{2}$-exposed pups than in those exposed to RA on d 3, 7, and 14 (Fig. 2C) (an effect of day and an effect of exposure). Although there was a developmental increase in liver GSSG levels from d 1 through 14 (an effect of day), no individual differences between treatment groups were detected by the statistical methods used (Fig. $2 D$ ). Our data indicated an effect of hyperoxic exposure on lung GSH/GSSG ratios with lower ratios observed in the hyperoxia-exposed groups than in the RA groups at $\mathrm{d} 3$ and 7 . No effects were indicated on liver GSH/GSSG ratios (data not shown).

As a marker of mitochondrial oxidative stress, CoASH and CoASSG levels were measured in tissue homogenates. No differences in lung CoASH levels were observed between exposure groups; however, lung CoASSG levels were greater at d 14 in hyperoxia-exposed pups than in RA-exposed controls (Fig. $3 A$ and $B$ ). In liver tissues, our analyses indicated an effect of day and an interaction be- 


\section{A}

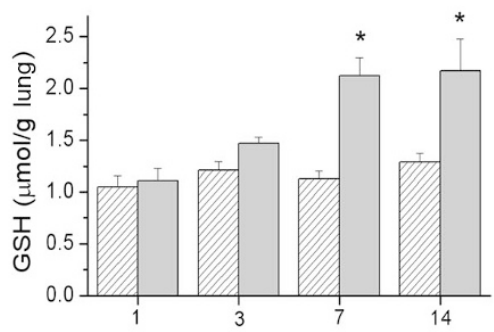

B
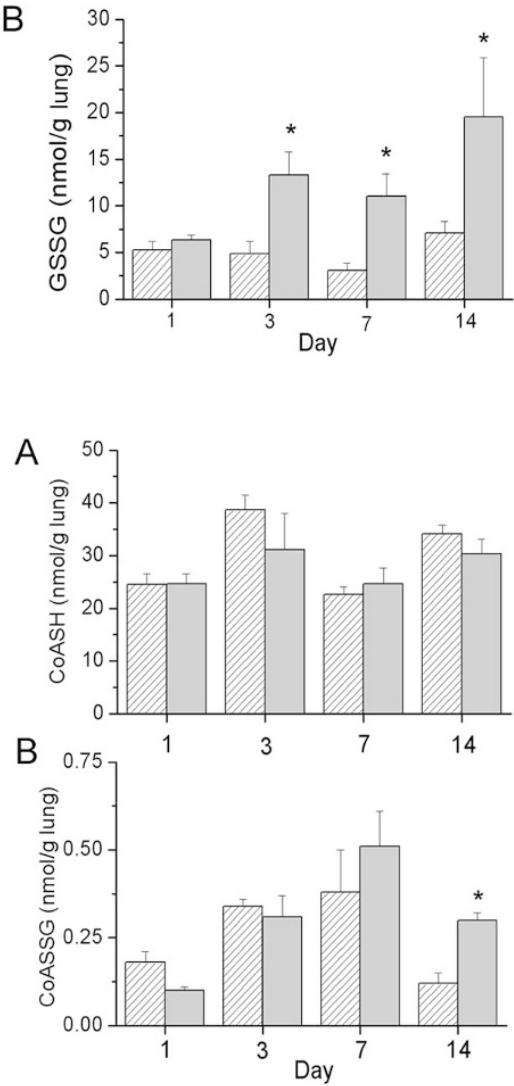
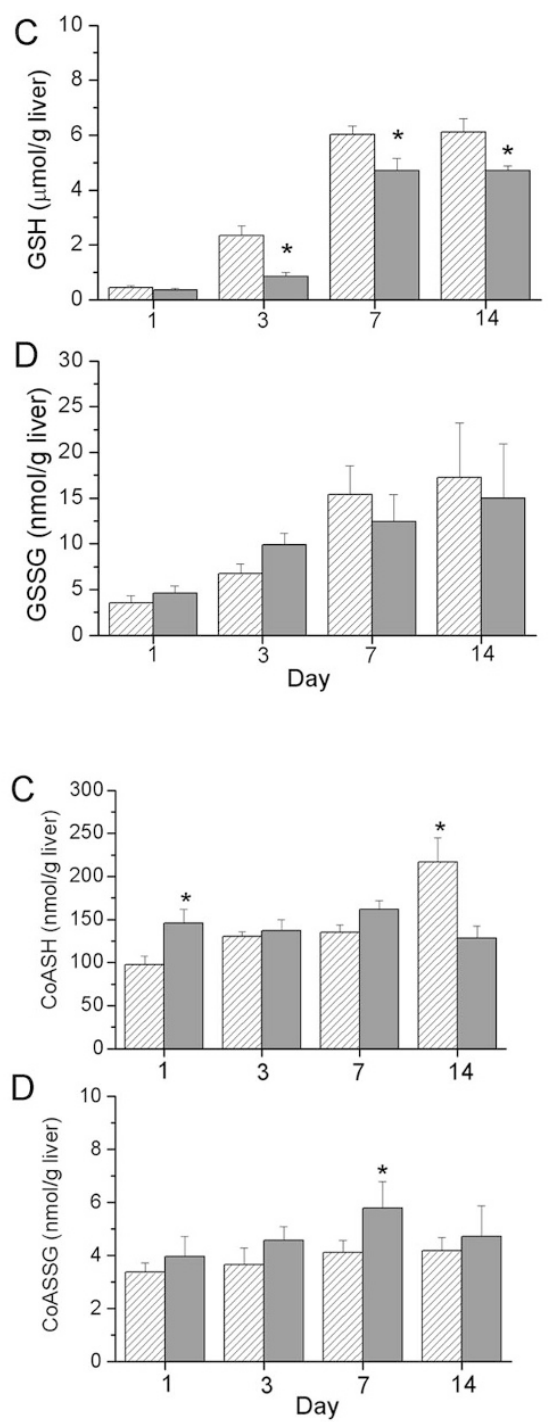

Figure 2. GSH and GSSG levels in lungs (A and $B)$ and livers $(C$ and $D)$ of newborn $\mathrm{C} 3 \mathrm{H} /$ HeN pups exposed to RA (white hatched bars) or $>95 \% \mathrm{O}_{2}$ (gray bars). Pups were killed at the designated times, and lung and liver tissues were snap frozen for analysis. GSH and GSSG levels were measured using the enzyme recycling assay as described in Methods. Results are reported as mean \pm SEM, and data were assessed by two-way ANOVA with modified $t$ test posthoc for individual differences, $n=5$ to 6 , $p<0.05$. An effect of exposure, an effect of day, and an interaction were detected on lung GSH and GSSG levels. An effect of day and an effect of exposure were detected on liver GSH levels and an effect of day was detected on liver GSSG levels. *Indicates different from same day RA.

Figure 3. CoASH and CoASSG levels in lungs $(A$ and $B)$ and livers $(C$ and $D)$ of newborn $\mathrm{C} 3 \mathrm{H} / \mathrm{HeN}$ pups exposed to RA (white hatched bars) or $>95 \% \mathrm{O}_{2}$ (gray bars). Pups were killed at the designated times, and lung and liver tissues were snap frozen for analysis. CoASH and CoASSG levels were measured by HPLC as described in Methods. Results are reported as mean \pm SEM and data were assessed by twoway ANOVA with modified $t$ test post hoc for individual differences, $n=3$ lung; $n=4$ to 7 liver, $p<0.05$. An effect of day was detected on CoASH and CoASSG levels in lung tissues. An effect of day and an interaction were detected on liver CoASH levels, and an effect of exposure was detected on liver CoASSG levels. *Indicates different from same day RA.
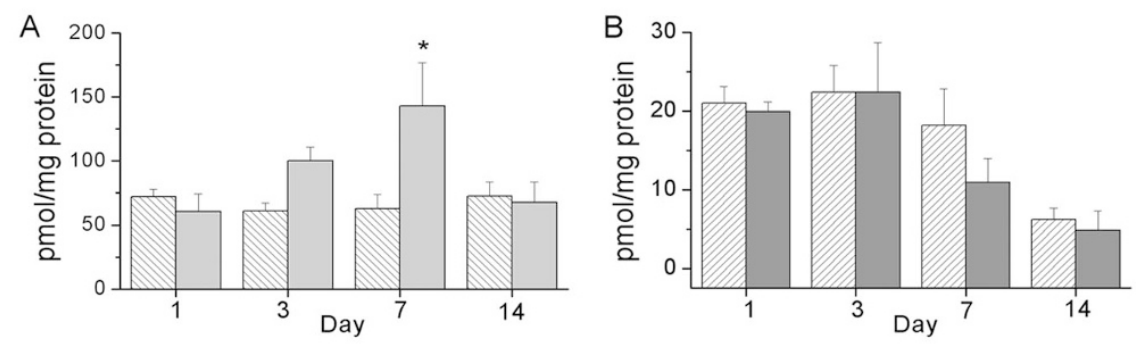

Figure 4. Nitrotyrosine levels in lung $(A)$ and liver $(B)$ tissues from newborn mouse pups exposed to RA (white hatched bars) or $>95 \% \mathrm{O}_{2}$ (gray bars). Snap-frozen tissues were homogenized and treated with dinitrophenyl hydrazine. Samples were hybridized to nitrocellulose membranes using a slot blot followed by incubation with anti-dinitrophenyl hydrazine antibodies as described in Methods. Immunoreactive bands were quantitated by densitometry. Results are reported as mean \pm SEM, and data were assessed by two-way ANOVA with modified $t$ test posthoc for individual differences, $n=5, p<0.05$. An effect of exposure and an interaction were detected on the lung tissues. An effect of day was detected on liver tissues. *Indicates different from same day RA.

tween exposure and day on CoASH levels. CoASH levels were greater in the $>95 \% \mathrm{O}_{2}$ exposed group than in RAexposed controls at $\mathrm{d} 1$ and were greater in RA controls than in hyperoxia-exposed pups at d 14 (Fig. 3C). An effect of exposure on CoASSG levels was detected and CoASSG levels were greater in the $>95 \% \mathrm{O}_{2}$-exposed pups than in the RA-exposed pups at $\mathrm{d} 7$ (Fig. $3 D$ ).
Nitrotyrosine levels were assessed in lung and liver homogenates as a marker of superoxide-mediated protein oxidation. An effect of exposure and an interaction between day and exposure was detected on nitrotyrosine levels in lung homogenates (Fig. 4A). Nitrotyrosine levels were greater at $\mathrm{d} 7$ in hyperoxia-exposed pups than in the RA controls. An effect of day was detected on liver nitrotyrosine levels and levels of 
nitrotyrosine tended to be lower in the liver homogenates obtained from hyperoxia-exposed pups than in those obtained from RA controls but no individual statistical differences were detected (Fig. 4B).

Lipoxygenases and cyclooxygenases, enzymes responsible for the production of hydroxyeicosatetraenoic acids (HETEs) and prostaglandins or thromboxane, respectively, are regulated by transcription factors that are responsive to changes in $\mathrm{O}_{2}$ tension. Our previous manuscript indicated greater levels of 5-LO and COX-2 at $\mathrm{d} 7$ and 14 in lung tissues of hyperoxiaexposed pups than those exposed to RA (22). In this study, our data indicate that immunoreactive 5-LO levels are greater in the livers of hyperoxia-exposed pups than in RA-exposed controls on days of life d 1 and 3 (Fig. 5A). Furthermore, immunoreactive hepatic COX-2 protein levels were greater in hyperoxia-exposed animals than in RA controls on days of life 3, 7, and 14 (Fig. 5B). These observations indicate that hepatic responses were detected at an earlier time point than similar responses in lung tissues.

HETEs can be formed by lipoxygenase activity or by free radical oxidation mechanisms. The concentrations of 5-, 8-, 9-, 11-, 12-, 15-HETE were measured independently. All HETE levels were similarly increased in the livers of the pups exposed to hyperoxia; however, for the purposes of this report, we have combined the individual measurements and have presented the data as total HETE levels. Our analyses indicated an effect of exposure on total HETE levels in the liver with subsequent analyses indicating greater HETE levels in the livers of hyperoxia-exposed pups than in RA-exposed controls at d 3 and 7 (Fig. 6A). These findings are in contrast with the lower levels of 5- HETE observed in lung tissues following hyperoxia exposure (22).

Similarly, the products of cyclooxygenases, specifically prostaglandins and thromboxane, were measured in liver tissues. For the purposes of these studies, we grouped prostaglandin $\mathrm{D}_{2}, \mathrm{E}_{2}, \mathrm{~F}_{2 \alpha}$, and thromboxane as a single measurement of cyclooxygenase products. Analysis indicated independent effects of day and exposure on the level of cyclooxygense products with greater levels at $\mathrm{d} 7$ in tissues from pups exposed to $>95 \% \mathrm{O}_{2}$ than in tissues obtained from RA controls, which are similar to the findings in the lung tissues from hyperoxia-exposed pups (22).

Histologic sections were analyzed on the livers tissues obtained from the pups exposed to RA or $>95 \% \mathrm{O}_{2}$. No pathology was observed including cholestasis, fibrosis, or acute inflammation. In addition, no changes in morphometry or cellular structure of the liver were indicated (data not shown).

\section{DISCUSSION}

The most striking findings in our studies in a newborn model of hyperoxic lung injury are the relatively early increases in 5-LO and COX-2 protein levels detected in the livers of hyperoxia-exposed animals (Fig. 5). In these studies, we measured tissue levels of these enzymes by immunoreac-
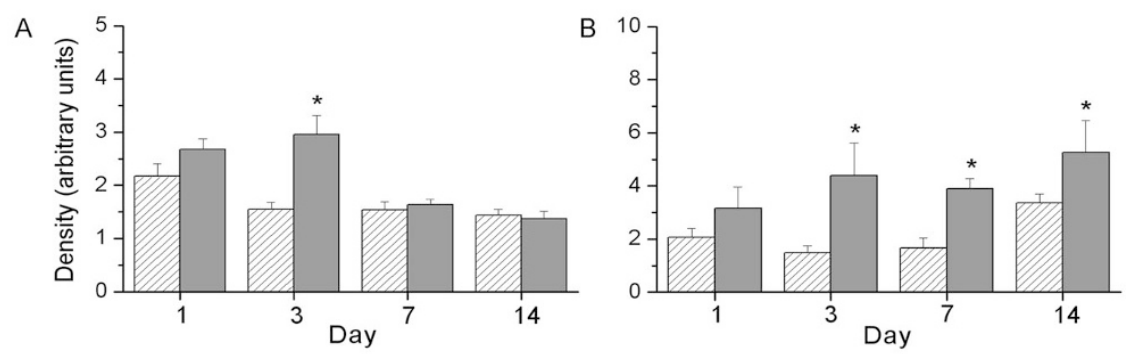

Figure 5. 5-LO and COX-2 protein levels in the liver tissue of newborn pups. Tissues were homogenized and proteins were separated by SDS-PAGE, transferred to nitrocellulose membranes, and probed with 5-LO $(A)$ or COX-2 $(B)$ antibodies. Bands were quantitated by densitometry, and data were normalized to $\beta$-actin. Results are reported as mean \pm SEM and data were assessed by two-way ANOVA with modified $t$ test posthoc for individual differences, $n=6$, $p<0.05$. And effect of day, an effect of exposure, and an interaction were detected on 5-LO levels, and an effect of exposure was detected on COX-2 levels. *Indicates different from same day RA.
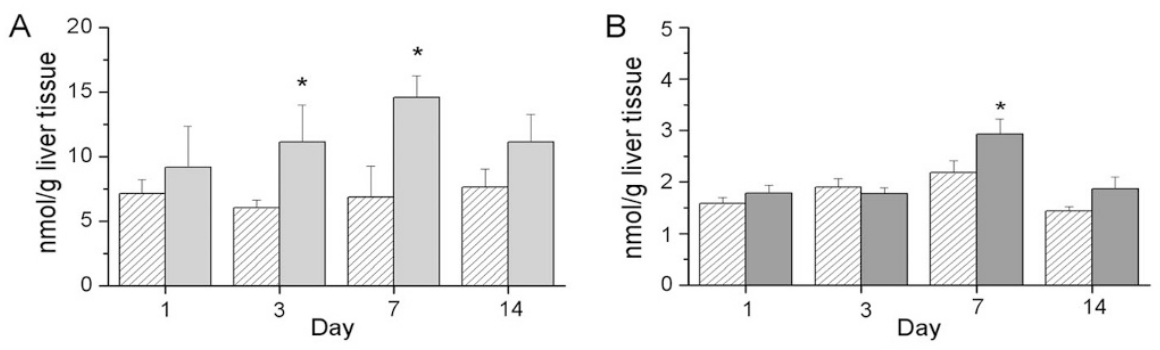

Figure 6. Lipid levels in liver tissues from newborn mouse pups exposed to RA (white hatched bars) or $>95 \% \mathrm{O}_{2}$ (gray bars). Snap-frozen tissues were extracted and lipids were analyzed by LC-MS/MS as described in Methods. Total HETE levels were the sum of 5-, 8-, 9-, 11-, and 12-HETE measurements (A). Total prostaglandin levels represent the sum of prostaglandin $\mathrm{D}_{2}, \mathrm{E}_{2}, \mathrm{~F}_{2 \alpha}$, and thromboxane $\mathrm{B}_{2}$ measurements $(B)$. Results are reported as mean \pm SEM, and data were assessed by two-way ANOVA with modified $t$ test post hoc for individual differences, $n=5$ to $7, p<0.05$. An effect of exposure was observed on total hepatic HETE levels, and an effect of day and an effect of exposure were observed on total prostaglandin levels. *Indicates different from same day RA. 
tivity. Therefore, the impact of these alterations on enzyme function cannot be determined. However, products of 5-LO, which contribute to the total HETE levels reported, were elevated in the liver in temporal association with the increase in immunoreactivity for 5-LO (Fig. 6A) suggesting that activity was also increased. Furthermore, total hepatic prostaglandins were also elevated in hyperoxia-exposed animals in temporal association with the increase in immunoreactivity for COX-2 (Figs. 5 and 6). Collectively, these data suggest that COX-2 and 5-LO mediate a functional response to hyperoxia in the liver.

Interestingly, increases in 5-LO and COX-2 protein levels occur in the livers of hyperoxia-exposed animals in the absence of a "classic" oxidant response as our data did not indicate appreciable effects of hyperoxic exposure on hepatic levels of GSSG, CoASSG, or nitrotyrosine. The observed decrease in hepatic GSH levels, which was detected early in the course of hyperoxic exposure, could be attributable to an increase in the formation of peroxides (Fig. 2C). If this were the case, however, a concomitant increase in GSSG would be expected (Fig. 2D). The lack of an observed increase in liver GSSG levels could be related to the capacity of the newborn mouse liver to actively export GSSG into the bile. The overall alterations of liver CoASSG levels in response to hyperoxic exposure were not remarkable and are not likely associated with the mechanisms of injury (Fig. 3).

Earlier data generated from our lab demonstrated a decrease in total GSH and GSSG in the livers of adult rodents exposed to hyperoxia $(11,23)$ similar to our present findings in newborn mice. The lack of evidence for mitochondrial-specific hepatic oxidant responses in a newborn model of hyperoxic lung injury is in contrast with previously reported studies in adult models. In adults, hepatic CoASSG levels were substantially elevated early in the course of hyperoxic exposure indicating mitochondrial compartmentalization (15). In our current studies, however, we observed only isolated minimal changes in the CoASH/CoASSG redox couple.

Lung oxidant responses in the present studies were markedly more pronounced than hepatic responses. Observed elevations in lung GSSG levels imply an increase in peroxide formation in response to hyperoxia, a decrease in the efficiency of GSSG export, or a reduction in GSH reductase activity (Fig. 2B). However, the lung tissues were not perfused because of their small size and any increase in red blood cells in the lung because of injury, could have contributed, at least in part, to the observed elevations in GSH and GSSG levels at 7 and $14 \mathrm{~d}$. The increase in lung GSSG contents in these studies occurs at the same time as the early influx of neutrophils (d 3) into the lungs of hyperoxia-exposed newborn mice (22). This finding argues against oxidation as a primary result of reactions attributable to inflammatory cell infiltration. Furthermore, our data on lung GSH and GSSG contents are also in contrast to our previously reported findings in adult hyperoxic lung injury models. Malleske et al. (11) and Tipple et al. (23) both reported that lung GSH and GSSG levels either remained unchanged or decreased in adult mice during the course of hyperoxic exposure. The lack of an appreciable effect of hyperoxic exposure on lung CoASH/CoASSG levels in the newborn mouse (Fig. 3) is also in contrast to previously published data in adult rat and mouse models of hyperoxic lung injury in which decreases in both CoASH and CoASSG were observed $(15,20)$. These findings clearly demonstrate that different mechanisms mediate responses to hyperoxic exposure in newborn and adult mice and that in this newborn model, oxidant stresses are mechanistically important.

Interestingly, nitrotyrosine measurements were increased in the lungs of hyperoxia-exposed pups (Fig. 4A) but were decreased in both RA- and hyperoxia-exposed liver tissues (Fig. 4B). In fact, our data indicated developmental decreases in liver nitrotyrosine contents independent of oxygen exposure. The greater nitrotyrosine levels in the lung tissues of hyperoxia-exposed pups indicate that superoxide production is increased in this model. The lack of nitrotyrosine accumulation in liver tissues obtained from hyperoxia-exposed pups further suggests the lack of excessive peroxynitrite formation in the liver. These findings, in addition to greater lung GSSG levels in the hyperoxia-exposed pups, provide evidence that the dominant effect of hyperoxia in the lungs of newborn pups is mediated through free radical oxidation. However, free radical oxidation mediated through peroxide or superoxide formation does not seem to be evident in the liver tissues.

The mechanism responsible for the induction of COX-2 and 5-LO in the liver is unclear from these data. Histologic analyses indicated no evidence of acute liver inflammation, fibrosis, or cholestasis (data not shown) providing evidence that the increase in lipid metabolizing enzymes and their products are not the result of direct liver injury. Existing literature suggests that these enzymes may be induced by an oxidant stress or by an increased availability of lipid substrates $(24,25)$. The lack of an appreciably detectable oxidant response in the livers of pups exposed to hyperoxia argues against these enzymes being induced in response to an accumulation of reactive oxygen species. Therefore, we speculate that the likely mechanism of induction of COX-2 and 5-LO is facilitated by accumulation of lipid substrates.

The constellation of findings in this report is consistent with a series of events in the lung and the liver that could contribute to total body responses to hyperoxia. In the lung, early responses to hyperoxic exposure are best characterized as primarily oxidant; whereas in the liver, early findings are best characterized by alterations in lipid metabolism. The observed alterations in protein levels of lipid metabolizing enzymes COX-2 and 5-LO were associated with significant changes in the liver lipid profiles in these studies. One speculation would be that if secreted into the systemic circulation, the products of these enzymes, specifically HETEs, could contribute to the observed inflammation and lung growth arrest observed. However, because of sample size limitations in newborn mice, larger animal models will be required to investigate this possibility.

In human infants, the lung epithelium is exposed to hyperoxia when supplemental oxygen is used to support patients with lung disease. Because of the insufficiency of lung function, other cells and organs should not be directly exposed to hyperoxia as they are in animal models of hyperoxia exposure. However, reports in human studies of increased oxidation, 
increased lung inflammation, and developmental arrest in infants developing bronchopulmonary dysplasia are similar to the findings in animal studies suggesting similar mechanisms are involved, despite differences in arterial pressure and arterial $\mathrm{CO}_{2}$ levels. If these data can be extrapolated to premature infants, further delineation of the mechanisms responsible for the observed findings could lead to interdictions designed to prevent the development of lung disease in this vulnerable population.

\section{REFERENCES}

1. Jobe AH, Bancalari E 2001 Bronchopulmonary dysplasia. Am J Respir Crit Care Med 163:1723-1729

2. Jobe AH 2005 Antenatal associations with lung maturation and infection. J Perinatol 25:S31-S35

3. Viscardi RM, Muhumuza CK, Rodriguez A, Fairchild KD, Sun CC, Gross GW, Campbell AB, Wilson PD, Hester L, Hasday JD 2004 Inflammatory markers in intrauterine and fetal blood and cerebrospinal fluid compartments are associated with adverse pulmonary and neurologic outcomes in preterm infants. Pediatr Res 55:1009-1017

4. Clark RH, Gerstmann DR, Jobe AH, Moffitt ST, Slutsky AS, Yoder BA 2001 Lung injury in neonates: causes, strategies for prevention, and long-term consequences. J Pediatr 139:478-484

5. Groneck P, Gotze-Speer B, Oppermann M, Eiffert H, Speer CP 1994 Association of pulmonary inflammation and increased microvascular permeability during the development of bronchopulmonary dysplasia: a sequential analysis of inflammatory mediators in respiratory fluids of high-risk preterm neonates. Pediatrics 93:712-718

6. Jobe AH, Ikegami M 1998 Mechanisms initiating lung injury in the preterm. Early Hum Dev 53:81-94

7. Speer CP 2006 Inflammation and bronchopulmonary dysplasia: a continuing story. Semin Fetal Neonatal Med 11:354-362

8. Ryan RM, Ahmed Q, Lakshminrusimha S 2008 Inflammatory mediators in the immunobiology of bronchopulmonary dysplasia. Clin Rev Allergy Immunol 34:174-190

9. Krowka MJ, Wiseman GA, Burnett OL, Spivey JR, Therneau T, Porayko MK, Wiesner RH 2000 Hepatopulmonary syndrome: a prospective study of relationships between severity of liver disease, $\mathrm{PaO}(2)$ response to $100 \%$ oxygen, and brain uptake after (99m)Tc MAA lung scanning. Chest 118:615-624

10. Fallon MB 2005 Mechanisms of pulmonary vascular complications of liver disease: hepatopulmonary syndrome. J Clin Gastroenterol 39:S138-S142

11. Malleske DT, Rogers LK, Velluci SM, Young TL, Park MS, Long DW, Welty SE, Smith CV, Nelin LD 2006 Hyperoxia increases hepatic arginase expression and ornithine production in mice. Toxicol Appl Pharmacol 215:109-117

12. Miralles C, Agusti AG, Aubry C, Sanchez JC, Walzer C, Hochstrasser D, Busquets X 2000 Changes induced by oxygen in rat liver proteins identified by high-resolution two-dimensional gel electrophoresis. Eur J Biochem 267:5580-5584

13. Moorthy B, Nguyen UT, Gupta S, Stewart KD, Welty SE, Smith CV 1997 Induction and decline of hepatic cytochromes P4501A1 and 1A2 in rats exposed to hyperoxia are not paralleled by changes in glutathione S-transferase-alpha. Toxicol Lett 90:67-75

14. Couroucli XI, Welty SE, Geske RS, Moorthy B 2002 Regulation of pulmonary and hepatic cytochrome P4501A expression in the rat by hyperoxia: implications for hyperoxic lung injury. Mol Pharmacol 61:507-515

15. Wong YL, Smith CV, McMicken HW, Rogers LK, Welty SE 2001 Mitochondrial thiol status in the liver is altered by exposure to hyperoxia. Toxicol Lett 123:179193

16. Frank L, Bucher JR, Roberts RJ 1978 Oxygen toxicity in neonatal and adult animals of various species. J Appl Physiol 45:699-704

17. Frank L, Sosenko IR 1987 Prenatal development of lung antioxidant enzymes in four species. J Pediatr 110:106-110

18. Rogers LK, Smith CV 2003 Coenzyme A and coenzyme A-glutathione disulfide measurements by HPLC. Curr Protocol Toxicol 6.8:1-6

19. Mihm MJ, Yu F, Reiser PJ, Bauer JA 2003 Effects of peroxynitrite on isolated cardiac trabeculae: selective impact on myofibrillar energetic controllers. Biochimie 85:587-596

20. O'Donovan DJ, Rogers LK, Kelley DK, Welty SE, Ramsay PL, Smith CV 2002 CoASH and CoASSG levels in lungs of hyperoxic rats as potential biomarkers of intramitochondrial oxidant stresses. Pediatr Res 51:346-353

21. Rogers LK, Valentine CJ, Szczpyka M, Smith CV 2000 Effects of hepatotoxic doses of acetaminophen and furosemide on tissue concentrations of CoASH and CoASSG in vivo. Chem Res Toxicol 13:873-882

22. Rogers LK, Tipple TE, Nelin LD, Welty SE 2009 Differential responses in the lungs of newborn mouse pups exposed to $85 \%$ or $>95 \%$ oxygen. Pediatr Res $65: 33-38$

23. Tipple TE, Welty SE, Rogers LK, Hansen TN, Choi YE, Kehrer JP, Smith CV 2007 Thioredoxin-related mechanisms in hyperoxic lung injury in mice. Am J Respir Cell Mol Biol 37:405-413

24. Dennis EA 2000 Phospholipase A2 in eicosanoid generation. Am J Respir Crit Care Med 161:S32-S35

25. Fitzpatrick FA, Soberman R 2001 Regulated formation of eicosanoids. J Clin Inves 107:1347-1351 